\title{
Radiation Recall Supraglottitis in a Child
}

\author{
Brian J. Wiatrak, MD and Charles M. Myer III, MD
}

The recrudescence and modification of radiation effects precipitated by the use of chemotherapeutic agents has been reported extensively in the literature. The recall of quiescent radiation effects such as dermatitis, enteritis, esophagitis, pneumonitis, and mucositis has been most commonly associated with dactinomycin (actinomycin D) and doxorubicin (Adriamycin, Adria Laboratories, Columbus, $\mathrm{OH}$ ) therapy, although other chemotherapeutic agents have been implicated. Radiation recall supraglottitis has been reported previously in a 47-year-old adult with diffuse histiocytic lymphoma. We report the first case of radiation recall supraglottitis involving dactinomycin therapy in a child.

\section{CASE REPORT}

A 4-year-old white female initially presented at 2 years of age with a rapidly enlarging mass in the right neck and right peripharyngeal space. Incisional biopsy at that time revealed pleomorphic rhabdomyosarcoma, and a tracheotomy was performed because of obstructive apnea. A complete evaluation consisting of chest computed tomography scan, bone scan, lumbar puncture, and bone marrow biopsy revealed no evidence of systemic metastases or central nervous system involvement. The initial cervical computed tomography scan revealed a large right-sided peripharyngeal mass with areas of focal calcification and enhancement with contrast infusion (Fig 1). The tumor was graded as group III according to the Intergroup Rhabdomyosarcoma Study I protocol (gross residual tumor) and the patient was begun on a therapeutic protocol consisting of dactinomycin, ifosfamide, and vincristine. The tumor failed to resolve completely and, in January 1989, a resection was performed for residual disease in the neck and peripharyngeal space. The final pathology of the specimen was consistent with pleomorphic rhabdomyosarcoma. Postoperatively, the patient received $60 \mathrm{~Gy}$ of hyperfractionated radiation therapy. A second, 2-week course of chemotherapy consisting of ifosfamide and

Received September 14, 1990 from the Department of Otolaryngology, Section of Pediatric Otolaryngology, University of Michigan, Ann Arbor, Ml. Accepted for publication April 15, 1991.

Address correspondence and reprint requests to Brian J. Wiatrak, MD, 2451 Taubman Center, Box 0313, 1500 E Medical Center Dr, Ann Arbor, MI 48109-0312.

Copyright 1991 by W.B. Saunders Company

0196-0709/91/1204-0005\$5.00/0 vincristine was initiated 1 month postoperatively. Three months postoperatively, the patient underwent microlaryngoscopy and bronchoscopy, which revealed a normal larynx with a small amount of inflammation in the posterior glottis. The subglottis was completely normal. The patient was subsequently decannulated without difficulty.

One week after decannulation, the patient received further chemotherapy consisting of ifosfamide and vincristine and was discharged from the hospital. Four months after decannulation, she received further chemotherapy consisting of dactinomycin,vincristine, and ifosfamide. Soon after this course of chemotherapy, the patient developed increased airway obstruction with intermittent stridor. Her airway was felt to be stable with mild oxygen desaturation and was closely monitored at home with a pulse oximeter applied during sleep.

As part of routine tumor surveillance, a magnetic resonance imaging scan of the neck was obtained (Fig 2). A markedly decreased supraglottic airway was noted. Increased signal intensity on T2 images of the supraglottic

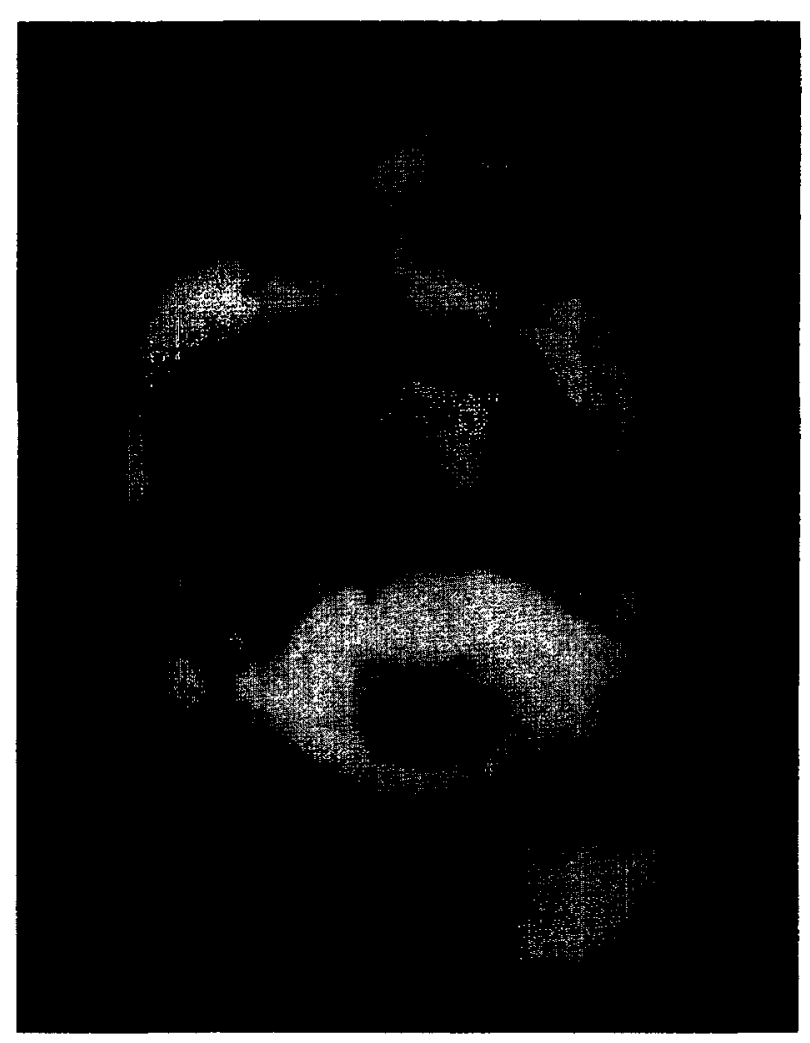

Figure 1. Computed tomography scan of a large, right-sided peripharyngeal rhabdomyosarcoma. 


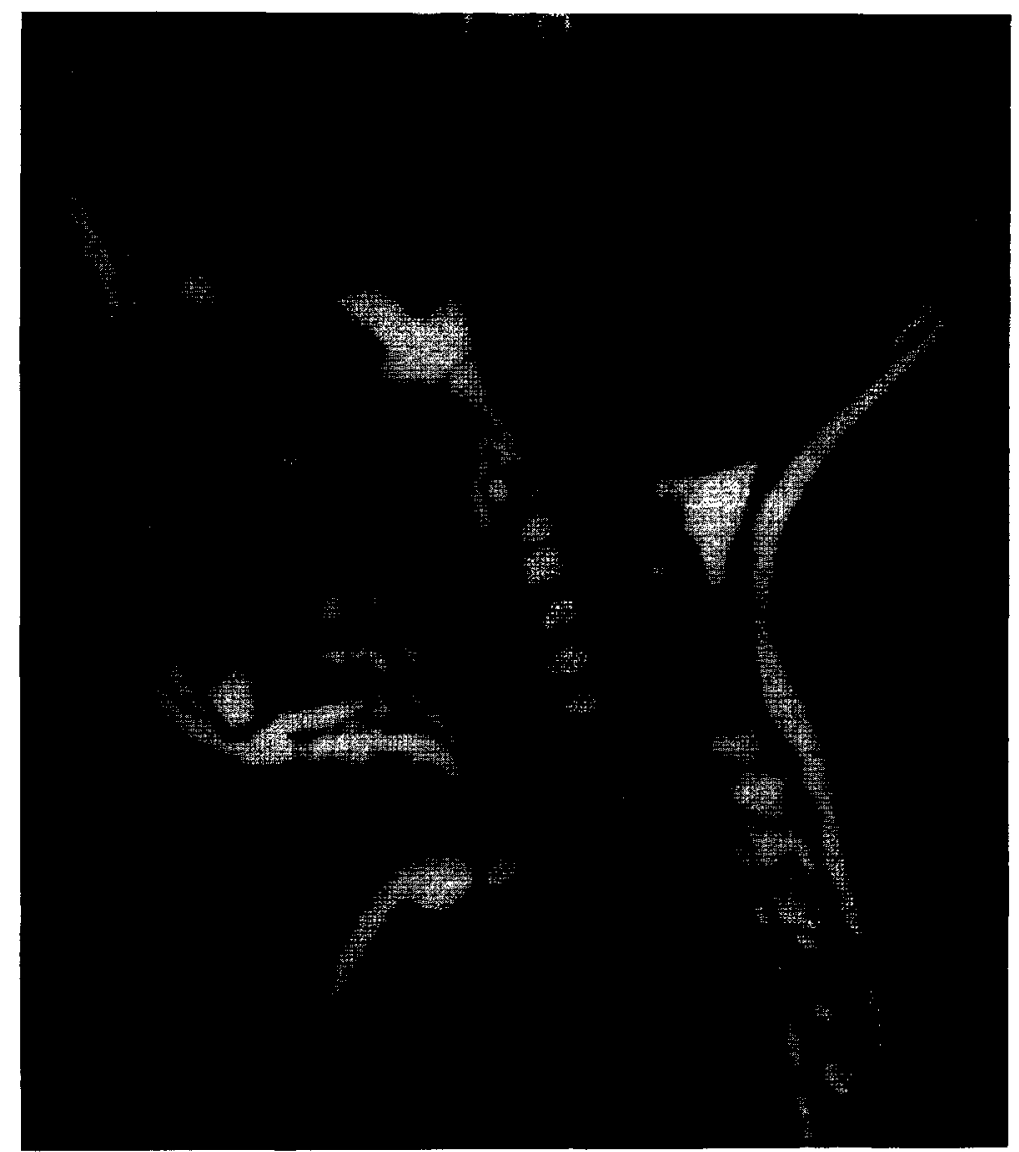

Figure 2. Magnetic resonance imaging scan of the neck after the administration of dactinomycin. Notice the fatty infiltration of cervical vertebrae 1-4 on the T1-weighted image, consistent with postradiation changes. The significant edema noted in the supraglottic airway lies within the previous radiation ports.

structures suggested edema rather than tumor infiltration. In addition, increased signal intensity in vertebral bodies 1 to 5 coincided with the previous radiation therapy ports. The supraglottic larynx was noted to lie within the previous radiation port. The patient was readmitted to the hospital at this time for microlaryngoscopy and bronchoscopy, which revealed marked epiglottic and supraglottic edema (Fig 3, left). The glottis could not be identified. Under mask ventilation, a tracheotomy was performed without difficulty.

Posttracheotomy laryngoscopy revealed a normal glottis (Fig 3, right). An epiglottic biopsy was obtained at this time and demonstrated inflammatory reaction. Microlaryngoscopy and bronchoscopy, performed 1 month later, revealed persistence of the supraglottic edema, which was, however, decreased from the previ-
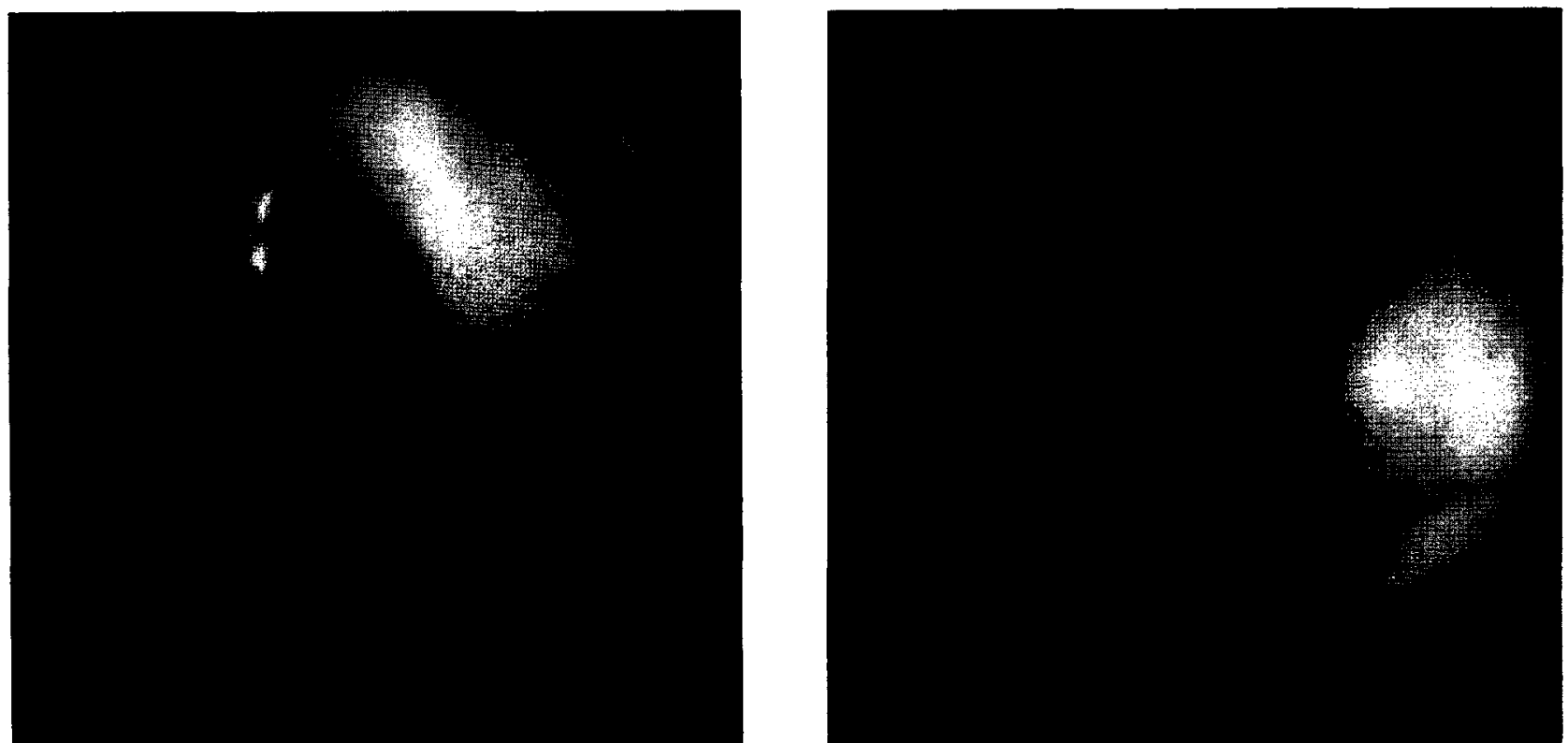

Figure 3. (Left) Endoscopic appearance of supraglottis after administration of dactinomycin. (Right) The true vocal cords remain normal in appearance. 
ous examination. Over the next year, the swelling in the supraglottic region decreased enough to allow decannulation. Subsequently, a stoma closure was required, but the child remains decannulated with normal exercise tolerance and minimal residual supraglottic edema 9 months following decannulation.

\section{DISCUSSION}

The augmentation of radiation reactions with the concurrent administration of chemotherapeutic agents is a well-described phenomenon most commonly associated with the antineoplastic antibiotics dactinomycin and doxorubicin. ${ }^{1-4}$ This phenomenon has been reported with the concurrent use of chemotherapeutic agents in most subclasses, including alkalating agents, cytotoxic agents, antineoplastic antibiotics, antimytotics, antimetabolites, and structural analogs. With the use of certain chemotherapeutic agents after the completion of radiotherapy, a recall phenomenon has been observed whereby the administration of a chemotherapeutic agent precipitates radiation tissue reaction within the previous radiation ports. ${ }^{5-9}$

Various organ systems may be involved in radiation recall toxicity. Reported radiation recall effects include radiation dermatitis, mucositis, esophagitis, pneumonitis, phlebitis, enteritis, and supraglottitis in an adult. ${ }^{1,5,6-10}$ The severity of the recall reaction is variable, but is probably related to the dosage of both the radiation and the drug. Other factors such as individual susceptibility, nutrition, and the timing of the administration of the drug relative to the radiation therapy also may play a role. ${ }^{1,11,12}$

Treatment of radiation recall reactions consists of the administration of corticosteroids, which often leads to a dramatic response. Response to dexamethasone has been widely reported. ${ }^{5,9,13}$ This treatment, however, was not appropriate in our patient since the tracheotomy bypassed the obstructive region.

Cessation of the offending chemotherapeutic agent also may decrease the severity of the recall reaction. It is interesting to note that our patient developed airway obstruction after the first dose of dactinomycin, which was administered after decannulation. She undoubtedly had supraglottic edema during the radiation therapy and possibly during the subsequent administrations of dactinomycin, which would not have been diagnosed due to the presence of the tracheotomy. Laryngoscopy and neck radiographs are not routinely obtained during radiation and chemotherapy.

The treatment for radiation recall supraglottitis consists of corticosteroids in the acute phase to decrease the amount of inflammation. Tissue response to steroids administered weeks to months after the initial insult is questionable. Surgical intervention should consist of tracheotomy in cases in which severe airway obstruction occurs. Conservative epiglottectomy may be considered, using sharp dissection or a $\mathrm{CO}_{2}$ laser to "debulk" the obstructing supraglottic structures. Aggressive resection in this area may result in postoperative aspiration, leading to long-term management problems. The role of supraglottic laryngectomy has not been discussed in the literature on the management of this particular problem. Our feeling is that such radical irreversible surgery places the patient at increased risk for aspiration. This procedure may be considered in adults who have adequate pulmonary reserve and understand the risks of aspiration associated with supraglottic laryngectomy.

\section{CONCLUSION}

This is the first reported case of radiation recall supraglottitis in a pediatric patient, attributed to the administration of dactinomycin for a rhabdomyosarcoma of the neck and peripharyngeal space. With numerous new chemotherapeutic agents being used to treat rhabdomyosarcoma and other childhood sarcomas as an adjuvant with radiation therapy, it is important to realize that radiation toxicity may be augmented by the concurrent administration of numerous chemotherapeutic agents or may recur at subsequent administrations as a recall phenomenon.

\section{References}

1. Phillips TL, Fu KK: Quantification of combined radiation therapy and chemotherapy effects on critical normal tissues. Cancer 1976; $37: 1186-1200$

2. Cassady JR, Richter MP, Piro AJ, et al: RadiationAdriamycin interactions: Preliminary clinical observations. Cancer 1975; 36:946-949

3. D'Angio GJ, Farber S, Maddock CL: Potentiation of x-ray effects by actinomycin-D. Radiology $1959 ; 73: 175-177$

4. Phillips TL, Wharam MD, Margolis LW: Modification of radiation injury to normal tissues by chemotherapeutic agents. Cancer 1975; 35:1678-1684

5. Wallenborn PA III, Postma DS: Radiation recall supraglottitis. Arch Otolaryngol 1984; 110:614-617

6. Stein RS: Radiation recall enteritis after actinumycin-D and Adriamycin therapy. South Med J 1978; 71:960-961

7. Sears ME: Erythema in areas of previous irradiation in patients treated with hydroxyurea. Cancer Chemother Rep $1964 ; 40: 31-32$

8. Newburger PE, Cassady RI, Jaffe N: Esophagitis due to Adriamycin and radiation therapy for childhood malignancy. Cancer 1978; 42:417-423

9. McInerney DP, Bullimore J: Reactivation of radiation pneumonitis by Adriamycin. Br J Radiol 1977; 50:224-227

10. Cassady IF, Richter M, Piro AI, et al: RadiationAdriamycin interactions: Preliminary clinical observations. Cancer 1975; 36:946-949

11. Vallejo $A$, McCormick B, Wittes $R$, et al: Combined therapy effect on normal tissue in the head and neck region. Front Radiat Ther Oncol 1979; 13:48-55

12. Fu KK: Normal tissue effects of combined radiotherapy and chemotherapy for head and neck cancer. Front Radiat Ther Oncol 1979; 13:113-132

13. Phillips TL. Wharamn MD, Margnlis LW: Modification of radiation injury to normal tissues by chemotherapeutic agents. 1975; Cancer 35:1678-1684 